DRAFT VERSION JANUARY 8, 2018

Preprint typeset using LATEX style AASTeX6 v. 1.0

\title{
ENVIRONMENTAL DEPENDENCE OF TYPE IA SUPERNOVA LUMINOSITIES FROM A SAMPLE WITHOUT A LOCAL-GLOBAL DIFFERENCE IN HOST STAR FORMATION
}

\author{
Young-Lo Kim ${ }^{1}$, Mathew Smith², Mark Sullivan ${ }^{2}$, And Young-Wook LeE ${ }^{1}$ \\ ${ }^{1}$ Center for Galaxy Evolution Research and Department of Astronomy, Yonsei University, Seoul 03722, Korea; ylkim83@yonsei.ac.kr, \\ ywlee2@yonsei.ac.kr \\ ${ }^{2}$ Department of Physics and Astronomy, University of Southampton, Southampton SO17 1BJ, UK
}

\begin{abstract}
It is now established that there is a dependence of the luminosity of type Ia supernovae (SNe Ia) on environment: SNe Ia in young, star-forming, metal-poor stellar populations appear fainter after light-curve shape corrections than those in older, passive, metal-rich environments. This is accounted for in cosmological studies using a global property of the SN host galaxy, typically the host galaxy stellar mass. However, recent low-redshift studies suggest that this effect manifests itself most strongly when using the local star-formation rate (SFR) at the SN location, rather than the global SFR or stellar mass of the host galaxy. At high-redshift, such local SFRs are difficult to determine; here, we show that an equivalent 'local' correction can be made by restricting the SN Ia sample in globally star-forming host galaxies to a low-mass host galaxy subset $\left(\leq 10^{10} M_{\odot}\right)$. Comparing this sample of $\mathrm{SNe}$ Ia (in locally star-forming environments) to those in locally passive host galaxies, we find that SNe Ia in locally star-forming environments are $0.081 \pm 0.018$ mag fainter $(4.5 \sigma)$, consistent with the result reported by Rigault et al. (2015), but our conclusion is based on a sample $\sim 5$ times larger over a wider redshift range. This is a larger difference than when splitting the SN Ia sample based on global host galaxy SFR or host galaxy stellar mass. This method can be used in ongoing and future high-redshift SN surveys, where local SN Ia environments are difficult to determine.
\end{abstract}

Keywords: cosmology: observations — distance scale — supernovae: general

\section{INTRODUCTION}

The most direct evidence for the accelerating universe is provided by distances inferred from the measurement of type Ia supernovae (SNe Ia; Riess et al. 1998; Perlmutter et al. 1999). The fundamental idea behind the use of SNe Ia is that their luminosities can be empirically standardized (Phillips 1993; Tripp 1998), and that standardization does not evolve with redshift or SN environment. Although this assumption was initially supported by small samples of SNe Ia and their host galaxies, which showed no clear luminosity dependence on host galaxy morphology (Riess et al. 1998; Schmidt et al. 1998; Perlmutter et al. 1999; Sullivan et al. 2003), more recent studies with larger numbers of SNe Ia have revealed subtle trends between the SN luminosity after empirical light-curve shape and color/extinction corrections, and host galaxy stellar mass, star formation rate (SFR) and specific SFR (sSFR; the SFR per unit stellar mass), and gas-phase metallicity (e.g., Kelly et al. 2010; Lampeitl et al. 2010; Sullivan et al. 2010; D'Andrea et al. 2011; Childress et al. 2013; Johansson et al. 2013; Pan et al. 2014).

In particular, the dependency of the Hubble residual on the host galaxy stellar mass $\left(M_{\text {stellar }}\right)$ is well-established: in the recent Joint Lightcurve Analysis (JLA) compilation of 740 SNe Ia (Betoule et al. 2014), SNe Ia in galaxies with $M_{\text {stellar }} \leq 10^{10} M_{\odot}$ were shown to be $0.061 \pm 0.012 \mathrm{mag}$ fainter than SNe Ia in galaxies with $M_{\text {stellar }}>10^{10} M_{\odot}$, after light curve shape and color corrections. The effect presumably arises because of differing properties of the progenitor stellar populations; the leading candidates are the progenitor age and the progenitor metallicity (Timmes et al. 2003; Kasen et al. 2009), both correlate with host galaxy stellar mass (e.g., Tremonti et al. 2004; Gallazzi et al. 2005; Kang et al. 2016).

These studies were based on measurements of the global properties of the SN Ia host galaxies, with the implicit assumption that the stellar population from which the SN progenitor originated shared these global properties. Although this assumption may be statistically true for large samples of objects, local environmental measurements at the SN location are likely to be more directly linked to the SN progenitor stellar populations. Such local environmental 
measurements of stellar age or metallicity are, however, difficult to make, and usually require dedicated spectroscopic programmes. One such example, Rigault et al. (2013, hereafter R13), used 82 spectroscopic measurements of the SFR at the SN position in the redshift range $0.03<z<0.08$, and found that SNe Ia in locally star-forming environments are $0.094 \pm 0.031 \mathrm{mag}$ fainter than those in locally passive environments. This result was reconfirmed by Rigault et al. (2015, hereafter R15) using an independent nearby sample of SNe Ia, where the local SFR was estimated instead from GALEX far-UV data; in total they estimate a magnitude offset of 0.094 \pm 0.025 mag (see also Roman et al. 2017 for a similar result from local $U-V$ color). However, Jones et al. (2015, hereafter J15) reached a different conclusion from the sample based on the same GALEX photometry, but including a very nearby sample $(0.01<z<0.023)$ and using an updated version of the Spectral Adaptive Lightcurve Template 2 (SALT2; Guy et al. 2007, 2010).

It is therefore important to be able to select sample of SNe Ia with known local galaxy properties, perhaps only based on global galaxy measurements, to clarify the effects of local environments on the SN Ia luminosities. The purpose of this paper is to show that such samples can be efficiently selected when the globally star-forming sample is restricted to relatively low mass hosts $\left(\leq 10^{10} \mathrm{M}_{\odot}\right)$, and that the environmental dependency of SN Ia luminosities is evident in this much larger sample. Only multi-band photometric data are used in this method, for which data for $\sim 1,000$ hosts are available in the literature from low to high redshift $(z<1.1)$.

\section{DATA}

The SNe Ia data used in this paper are drawn from the YOnsei Nearby Supernova Evolution Investigation (YONSEI) SN Catalog (Kim et al. 2015; Kang et al. 2016). This catalog is a superset of all SN Ia surveys adopted in the SNANA package (Kessler et al. 2009), containing 1059 SNe Ia over the redshift range $0.01<z<1.4$, including: low-redshift SN surveys (Hamuy et al. 1996; Riess et al. 1999; Jha et al. 2006; Hicken et al. 2009, 2012; Contreras et al. 2010; Stritzinger et al. 2011, hereafter 'Low-z'), the SDSS-II SN survey (Sako et al. 2014, hereafter SDSS), the ESSENCE survey (Miknaitis et al. 2007), the first three years of the Canada-France-Hawaii Telescope Supernova Legacy Survey (Guy et al. 2010, hereafter SNLS), and the Hubble Space Telescope sample of Riess et al. (2007). For the light-curve analysis, we re-fit all the original published light curves with the most up-to-date version of SALT2 (version 2.4 presented in Betoule et al. 2014), as implemented in the SNANA package. The Malmquist bias corrections are then applied to the sample. Because most of the Low- $z$, SDSS, SNLS, and HST samples in the JLA catalog are very similar to those in our catalog, we take the correction terms calculated by Betoule et al. (2014). For the ESSENCE sample, we adopt the correction values provided by Wood-Vasey et al. (2007). We then interpolate the bias correction value for each SN at given redshift, and this value is subtracted from all rest-frame peak apparent magnitude in $B$-band $\left(m_{B}\right)$. The YONSEI SN Catalog provides a rest-frame peak apparent magnitude in $B$-band $\left(m_{B}\right)$, a lightcurve shape parameter $\left(x_{1}\right)$, and a color parameter $(c)$ for each SN. In order to select only normal SNe Ia ${ }^{1}$ (the 'YONSEI Cosmology sample'), we apply various cuts to the sample based on the light-curve shape and color values $\left(-3<x_{1}<3\right.$ and $\left.-0.3<c<0.3\right)$, similar to those adopted in Betoule et al. (2014). Of the $1059 \mathrm{SNe}, 941$ pass this requirement. Finally, for our analyses of Hubble residuals and host properties, we restrict the SNLS sample to $z \leq 0.85$, as in Sullivan et al. (2010), where the SNLS SNe have the highest signal-to-noise, and the Malmquist corrections are smallest. Full details of our procedures will be published in a companion paper (Y.-L. Kim et al., in preparation).

For the purposes of this paper, which examines SNe Ia in the context of their host galaxies, we also require host galaxy information, specifically stellar masses and global sSFRs. For consistency across the samples, we use the PÉGASE.2 spectral synthesis code (Fioc \& Rocca-Volmerange 1997; Le Borgne et al. 2004) as described in detail in Sullivan et al. $(2006,2010)$. Briefly, we use a set of 14 exponentially declining star formation histories (SFHs) with SFR $\propto \exp ^{-t / \tau}$, where $t$ represents time and $\tau$ is the e-folding time. Each SFH has 100 time steps, and we use foreground dust screens ranging from $E(B-V)=0$ to $0.30 \mathrm{mag}$ in steps of 0.05. We fit the host galaxy data from Sullivan et al. (2010) (SNLS) and Smith et al. (2012) and Sako et al. (2014) (SDSS) using the above framework to ensure a consistent approach. 213 hosts for SNLS and 355 for SDSS are matched with the YONSEI Cosmology sample. For the Low- $z$ sample, 89 host information are taken from Neill et al. (2009) which uses the same PÉGASE.2 approach. In total, 657 SNe Ia and their host galaxies are collected for our analysis. The sample sizes and the data used in our analysis are listed in Tables 1 and 2, respectively.

Local host properties for 110 Low- $z$ samples are separately listed in Table 3. The local SFR surface density, global host class (e.g., star-forming or passive), and the probability that a SN has a locally passive environment $(\mathcal{P}(\mathrm{Ia} \epsilon))$ are taken from J15 and R152. Host stellar masses are collected from Neill et al. (2009) and Kelly et al. (2010).

\footnotetext{
${ }^{1}$ We discard peculiar, sub-, and over-luminous SNe, such as 1991bg-like and 2002cx-like, following the Betoule et al. (2014) scheme.
}

2 J15 showed a median offset in $\mathcal{P}(\mathrm{Ia} \epsilon)$ between J15 and R15 of $\sim 3 \%$. They showed that this has little impact on the final results. 
For the SALT2 model, the distance modulus for each SN is formed as

$$
\mu_{\mathrm{SN}}=m_{B}-M_{B}+\alpha \times x_{1}-\beta \times c
$$

where $\alpha, \beta$ and $M_{B}$ are nuisance parameters in the distance modulus estimate. Cosmological fits then minimise

$$
\chi^{2}=\sum_{\mathrm{SNe}} \frac{\mu_{\mathrm{SN}}-\mu_{\mathrm{model}}\left(z ; \Omega_{M}\right)}{\sigma_{\mathrm{stat}}^{2}+\sigma_{\mathrm{int}}^{2}}
$$

where $\mu_{\text {model }}\left(z ; \Omega_{M}\right)$ is the predicted distance modulus in the $\Lambda$ CDM cosmology we assume throughout this paper, $\sigma_{\text {stat }}$ is the statistical uncertainty for each $\mathrm{SN}$, and $\sigma_{\text {int }}$ is the so-called intrinsic dispersion added to the SN uncertainties to ensure a reduced $\chi^{2}\left(\chi_{\text {red }}^{2}\right.$; the $\chi^{2}$ per degree of freedom) of 1 . When we examine the systematic variation in SN Ia luminosity with the host galaxy properties, we set $\sigma_{\text {int }}=0$ (e.g., see D'Andrea et al. 2011; Gupta et al. 2011; Pan et al. 2014). We refer to the quantity $\mu_{\mathrm{SN}}-\mu_{\text {model }}\left(z ; \Omega_{M}\right)$ as the 'Hubble residual'. We use the JLA likelihood code (Betoule et al. 2014) to estimate baseline Hubble residuals for the SNe Ia. The best-fit cosmological parameters obtained from the YONSEI Cosmology sample were $\Omega_{M}=0.30, \alpha=0.15, \beta=3.69$, and $M_{B}=-19.06$ with $\sigma_{\text {int }}=0$. In the calculation of the weighted-mean of Hubble residuals described below, the error of the weighted-mean is corrected to ensure a $\chi_{\text {red }}^{2}=1$. We have also applied Chauvenet's criterion (Taylor 1997) to reject outliers during this procedure, removing 9 objects from our sample. For our analysis, this criterion corresponds to $3.1 \sigma$ on average.

For the Low- $z$ sample, the effect of peculiar velocities of the SN host galaxies relative to the Hubble flow may introduce a bias in the determination of the cosmological parameters. Our Low- $z$ sample uses the redshifts corrected for bulk flows (following Conley et al. 2011). Further, Dhawan et al. (2017) and Zhang et al. (2017) showed that this difference has only a negligible effect in measuring the Hubble constant $(\sim 0.4 \%)$. Consequently, we estimate the impact of peculiar velocities on our study - which is not focused at low-redshift - is likely to be very small.

\section{SELECTING SNE IA WITHOUT A LOCAL-GLOBAL DIFFERENCE IN STAR FORMATION}

R13 showed that SNe Ia occurring in globally passive host galaxies only occur in locally passive environments, but SNe Ia in globally star-forming host galaxies can occur in both locally star-forming and locally passive environments. Cleanly separating the latter class (we shall refer this as the local-global difference in star formation) into locally passive and locally star-forming subsets will clearly be of great benefit. For the globally star-forming host galaxies, we show in Figure 1 the local SFR surface density $\left(\log \left(\Sigma_{\mathrm{SFR}}\right)\right)$ as a function of the stellar mass $\left(M_{\text {stellar }}\right)$ for the Low- $z$ sample listed in Table 3. This figure shows that locally star-forming environments are found across a wide range of $M_{\text {stellar, }}$, while the locally passive environments are mostly found in the relatively massive galaxies. Dividing this figure into two regimes at $\log \left(M_{\text {stellar }}\right)=10$ gives a clear distinction between these two populations: in high-mass hosts $\left(\log M_{\text {stellar }}>10\right)$, SNe Ia can arise either from locally passive and locally star-forming environments; in low-mass hosts $\left(\log M_{\text {stellar }} \leq 10\right)$, SNe Ia arise only in locally star-forming environments. Thus, for globally star-forming host galaxies, a clean sample of SNe Ia in locally star-forming environments can be formed by selecting only host galaxies with $\log M_{\text {stellar }} \leq 10$.

With the assumption that this simple empirical criterion can be used for all globally star-forming hosts (with $\log (\mathrm{sSFR})>-10.4$ for our sample), we can apply this to all our globally star-forming hosts to select a sample without the local-global difference in star formation (i.e., in the locally star-forming environments). For the SNe Ia in globally passive hosts (with $\log (\mathrm{sSFR}) \leq-10.4$ ), we assume all are also in locally passive environments (as demonstrated by R13). This gives a final sample of 368 SNe Ia (out of 649 in Table 1) without the local-global difference in star formation, among which $194 \mathrm{SNe}$ Ia are in locally passive environments $\left(N_{\text {locally-passive }}=194\right)$, and $174 \mathrm{SNe}$ Ia are in locally star-forming environments $\left(N_{\text {locally }}-\mathrm{SF}=174\right)$.

\section{RESULTS}

The upper panel of Figure 2 shows the dependence of SN Ia luminosity on host galaxy properties from the sample without the local-global difference in star formation, as described in Section 3. We find that SNe Ia in locally starforming environments are fainter than those in locally passive environments: the difference in the weighted-mean of the Hubble residuals is $0.081 \pm 0.018 \mathrm{mag}$ (see Table 4). These values are comparable to the combined result of R15: $0.094 \pm 0.025 \mathrm{mag}(3.5 \sigma)$ for SALT2. Our result, however, is statistically more significant $(4.5 \sigma)$, as the present analysis is based on a $\sim 5$ times larger sample that covers a wider redshift range $(0.01<z \leq 0.85)$. This is an independent confirmation of the environmental dependency of SN Ia luminosity from the sample without the local-global difference in star formation - but based only on multi-band photometry. 
We contrast this result to that based on our full YONSEI Cosmology sample. The lower panel of Figure 2 also shows this cosmology sample as a function of sSFR, but including all SNe Ia in star-forming galaxies, regardless of their stellar mass. The sample of SNe Ia in globally star-forming galaxies with $\log M_{\text {stellar }}>10$ have more negative Hubble residuals (i.e., are brighter, $-0.009 \pm 0.010$ ) than those SNe Ia in globally star-forming galaxies with log $M_{\text {stellar }} \leq 10$ $(0.038 \pm 0.013)$. The difference in Hubble residual between globally star-forming and globally passive galaxies is reduced to $0.049 \pm 0.015 \mathrm{mag}$ (see Table 4 ). We also compare the results when splitting the sample according to $M_{\text {stellar }}$ (see Table 4). For such a sample, the difference in Hubble residual is not as large as the result from a sample without the local-global difference.

We check the probability of observing our main result by chance using a Monte Carlo permutation test. We randomly draw $N_{\text {locally-SF }}$ SNe Ia from our full star-forming sample (without replacement) and calculate the difference in Hubble residuals between this randomly selected star-forming sample and the globally passive/locally passive hosts. In 100,000 realizations, $\simeq 0.4 \%$ of the samples have a larger value for the difference in Hubble residuals than our main result.

As discussed by J15 and Rigault (2015), the redshift cut may affect their results, which show an apparent discrepancy (see Section 1). In order to investigate this effect, we have selected a sample overlapping in redshift with J15 $(0.010<$ $z<0.1)$ and R15 $(0.023<z<0.1)$. In the case of a sample overlapping with J15 (67 SNe), we also observe no significant environmental dependency in the SN luminosity: the luminosity differences are $0.011 \pm 0.042 \mathrm{mag}$. For the sample overlapping in redshift with R15 (43 SNe), the luminosity differences are $0.080 \pm 0.046 \mathrm{mag}(1.7 \sigma)$. These results are consistent, if at slightly lower significance than those of R15, who found differences at $2.5 \sigma$. For our full redshift sample only $\sim 6 \%$ of SNe have $z<0.023$, so the redshift cut has only negligible effect on our main result.

\section{IMPLICATION FOR COSMOLOGY}

The environmental dependency of SN Ia luminosity obtained in this paper is consistent with a link between SN Ia progenitor age and SN Ia standardised luminosity: those in passive environments, brighter after light-curve shape correction, and those in star-forming environments, fainter after light-curve shape correction. As the cosmic starformation history evolves sharply with redshift, the mix of these SNe Ia is also likely to change, with the fraction of SNe in locally passive environments most likely decreasing with increasing redshift (e.g. Sullivan et al. 2006). In order to predict the impact on cosmology, we have employed a simple model for the fraction of SNe Ia located in locally passive environments as a function of redshift, $\psi(z)$, from R13 (their Eq.(5)), defined as

$$
\psi(z)=\left(K \times 10^{0.95 z}+1\right)^{-1}
$$

with $K=0.90 \pm 0.15$ from the normalization of $\psi(z=0.05)=50 \pm 5 \%$, based on their observational data. The difference in Hubble residual between SNe in locally passive and those in locally star-forming environments as a function of redshift can then be written as

$$
\Delta \operatorname{HR}_{\mathrm{SFR}}(z)=A \times \psi(z)
$$

For the calibration of $\Delta \mathrm{HR}_{\mathrm{SFR}}(z)$, we split our data into three redshift bins with equal numbers of SNe $(z \leq 0.180$, $0.180<z<0.337$, and $0.337<z, \sim 123$ SNe in each bin), from which we measure $A$ to be $-0.204 \pm 0.035$. Figure 3 shows the evolution of environmental dependence of SN luminosity from these three redshift bins, which is compared with the simple evolution model from Eq. (4). The model is consistent with our observed data, similar to the study of R13, but that study is based on the mass-step evolution model $\left(\Delta M_{B \text {, mass }}^{\text {corr }}(z)\right.$, see their Figure 11$)$. Clearly, further SN Ia data are required to test the model in detail. R13 pointed out that ignoring any observed redshift evolution of the HR difference could shift the dark energy equation-of-state by $\Delta w \sim-0.06$; our result would suggest a similar shift in $w$.

The sample without the local-global difference in star formation can also give the more robust results when estimating cosmological parameters, in terms of the r.m.s. scatter of the Hubble residuals (Figure 2 and Table 4 ) and the intrinsic scatter (Table 5). Interestingly, SNe Ia in locally star-forming environments have a $2 \%$ smaller r.m.s. scatter, and also require a $5 \%$ smaller intrinsic scatter than those in the full local sample. This may indicate that this sample is made up of the most homogeneous sample in terms of progenitor ages, and therefore less affected by a possible luminosity evolution of SNe Ia.

\section{DISCUSSION}

We have shown that a sample without the local-global difference in star formation can be efficiently selected, when the globally star-forming sample is restricted to the relatively low-mass hosts $\left(\leq 10^{10} \mathrm{M}_{\odot}\right)$. By employing this technique, we find that SNe Ia in locally star-forming environments are $0.081 \pm 0.018$ mag fainter than those in locally passive 
environments, after light-curve shape and color corrections. When only the lowest redshift bin $(z \leq 0.180)$ is considered, this luminosity difference increases slightly to $0.091 \pm 0.031 \mathrm{mag}$. This is consistent with the results suggested by R13 and R15. Our results are, however, statistically more significant $(4.5 \sigma)$ than previous results, because our sample is $\sim 5$ times larger.

As noted above, a clear distinction in the local-global difference in star formation is observed when the sample is divided at $\log \left(M_{\text {stellar }}\right)=10$. The well-established mass-step in SN Ia luminosity also occurs near this host mass (Kelly et al. 2010; Lampeitl et al. 2010; Sullivan et al. 2010; Gupta et al. 2011; Childress et al. 2013; Betoule et al. 2014; Pan et al. 2014). Numerous studies pointed out the uniqueness of the mass scale of $10^{10} \mathrm{M}_{\odot}$. For example, Cappellari et al. (2013) and Bernardi et al. (2016) showed that $M_{\text {stellar }} \sim 3 \times 10^{10} M_{\odot}$ is related to a transition in the assembly histories of galaxies for both early- and late-type galaxies. Furthermore, Kauffmann et al. (2003), Balcells et al. (2007), and Hopkins et al. (2009) showed a transition of galaxy morphology, such that galaxy morphology is changed from late- to early-type or from a disk to a bulge-dominated system, occurs near this mass scale. These results suggest that the origin of luminosity difference between SNe Ia in star-forming (low-mass) and those in passive (high-mass) hosts may be related to these transitions, because the average mass, metallicity, and population age of hosts change as well. In particular, in the case of globally star-forming low-mass ( $\left.\log M_{\text {stellar }} \leq 10\right)$ galaxies, the star formation history is characterized by recent starbursts with little contribution from older stars. By contrast, in globally star-forming high-mass galaxies, the star formation history appears more extended, with more contributions from older stars (e.g., Kauffmann et al. 2003; Salim et al. 2007). Therefore, SNe Ia in globally star-forming low-mass hosts are more likely originating from young progenitors (see also figure 3 of Childress et al. 2014).

Our result on the luminosity difference between SNe Ia in locally passive and those in locally star-forming environments is qualitatively consistent with the well-established mass-step in SN Ia luminosity. Since the host mass and SFR cannot directly affect SN luminosity, many studies pointed out that this is most likely due to the population properties of a host galaxy, such as age and metallicity (Johansson et al. 2013; Childress et al. 2014; Pan et al. 2014; Graur et al. 2015; Kang et al. 2016). Specifically, Kang et al. (2016) found that stellar population age is mainly responsible for the relation between host mass and Hubble residual. This would imply that the properties of SN can vary with a mean population age of a host galaxy. As this quantity is known to evolve with redshift, the properties of a progenitor would also change with redshift. This in turn may affect the details of SN explosion mechanism, and therefore would lead to a possible luminosity evolution of SNe Ia, as highlighted in Figure 3. Since the luminosity evolution can cause a potential bias in the estimation of cosmological parameters, it deserves a careful consideration when using SN Ia for cosmological analyses.

Finally, we note that, even though $\sim 43 \%$ of the full local sample is not used after performing the analysis suggested in this paper, SNe Ia in locally star-forming environments could give more robust results: a $\sim 2 \%$ smaller r.m.s. scatter of the Hubble residual and a $\sim 5 \%$ smaller intrinsic scatter than when using the full local sample (see Section 5). As has also been suggested by Rigault et al. (2013), Childress et al. (2014), and Kelly et al. (2015), this homogeneous sample can also lead to the improved application of SNe Ia as cosmological distance indicators. Therefore, future SN Ia cosmology surveys should consider the measurements of the local environments of host galaxies. The local properties of hosts, however, cannot be directly determined from spectroscopy, even in the era of 30-m class telescopes. In this respect, the method presented in this paper, which requires only global multi-band host galaxy photometry, could be adopted in forthcoming high-redshift SN surveys.

We thank the referee for his/her careful reading of the manuscript and many helpful comments. Y.-L. K. thanks the Department of Physics and Astronomy at the University of Southampton for their hospitality during the visits, and Mario Pasquato for helpful suggestions on the MCMC test. M.S. acknowledges support from EU/FP7-ERC grant No. [615929]. Support for this work was provided by the National Research Foundation of Korea to the Center for Galaxy Evolution Research through the grant programs No. 2017R1A5A1070354 and 2017R1A2B3002919.

\section{REFERENCES}

Balcells, M., Graham, A. W., \& Peletier, R. F. 2007, ApJ, 665, 1104

Bernardi, M., Meert, A., Sheth, R. K., et al. 2016, MNRAS, 455, 4122

Betoule, M., Kessler, R., Guy, J., et al. 2014, A\&A, 568, A22

\author{
Cappellari, M., McDermid, R. M., Alatalo, K., et al. 2013, \\ MNRAS, 432, 1862 \\ Childress, M., Aldering, G., Antilogus, P., et al. 2013, ApJ, 770, \\ 108 \\ Childress, M. J., Wolf, C., \& Zahid, H. J. 2014, MNRAS, 445, \\ 1898
}


Conley, A., Guy, J., Sullivan, M., et al. 2011, ApJS, 192, 1

Contreras, C., Hamuy, M., Phillips, M. M., et al. 2010, AJ, 139, 519

D'Andrea, C. B., Gupta, R. R., Sako, M., et al. 2011, ApJ, 743, 172

Dhawan, S., Jha, S. W., \& Leibundgut, B. 2017, arXiv:1707.00715

Fioc, M., \& Rocca-Volmerange, B. 1997, A\&A, 326, 950

Gallazzi, A., Charlot, S., Brinchmann, J., White, S. D. M., \& Tremonti, C. A. 2005, MNRAS, 362, 41

Graur, O., Bianco, F. B., \& Modjaz, M. 2015, MNRAS, 450, 905

Gupta, R. R., D'Andrea, C. B., Sako, M., et al. 2011, ApJ, 740, 92

Guy, J., Astier, P., Baumont, S., et al. 2007, A\&A, 466, 11

Guy, J., Sullivan, M., Conley, A., et al. 2010, A\&A, 523, A7

Hamuy, M., Phillips, M. M., Suntzeff, N. B., et al. 1996, AJ, 112, 2408

Hicken, M., Challis, P., Jha, S., et al. 2009, ApJ, 700, 331

Hicken, M., Challis, P., Kirshner, R. P., et al. 2012, ApJS, 200, 12

Hopkins, P. F., Somerville, R. S., Cox, T. J., et al. 2009, MNRAS, 397, 802

Jha, S., Kirshner, R. P., Challis, P., et al. 2006, AJ, 131, 527

Johansson, J., Thomas, D., Pforr, J., et al. 2013, MNRAS, 435, 1680

Jones, D. O., Riess, A. G., \& Scolnic, D. M. 2015, ApJ, 812, 31 (J15)

Kang, Y., Kim, Y.-L., Lim, D., Chung, C., \& Lee, Y.-W. 2016, ApJS, 223, 7

Kasen, D., Röpke, F. K., \& Woosley, S. E. 2009, Nature, 460, 869

Kauffmann, G., Heckman, T. M., White, S. D. M., et al. 2003, MNRAS, 341, 54

Kim, Y.-L., Kang, Y., \& Lee, Y.-W. 2015, Publication of Korean Astronomical Society, 30, 485

Kelly, P. L., Filippenko, A. V., Burke, D. L., et al. 2015, Science, 347, 1459

Kelly, P. L., Hicken, M., Burke, D. L., Mandel, K. S., \& Kirshner, R. P. 2010, ApJ, 715, 743

Kessler, R., Bernstein, J. P., Cinabro, D., et al. 2009, PASP, 121, 1028

Lampeitl, H., Smith, M., Nichol, R. C., et al. 2010, ApJ, 722, 566

Le Borgne, D., Rocca-Volmerange, B., Prugniel, P., et al. 2004, A\&A, 425, 881

Miknaitis, G., Pignata, G., Rest, A., et al. 2007, ApJ, 666, 674

Neill, J. D., Sullivan, M., Howell, D. A., et al. 2009, ApJ, 707, 1449
Pan, Y.-C., Sullivan, M., Maguire, K., et al. 2014, MNRAS, 438, 1391

Perlmutter, S., Aldering, G., Goldhaber, G., et al. 1999, ApJ, 517, 565

Phillips, M. M. 1993, ApJL, 413, L105

Riess, A. G., Filippenko, A. V., Challis, P., et al. 1998, AJ, 116, 1009

Riess, A. G., Kirshner, R. P., Schmidt, B. P., et al. 1999, AJ, 117,707

Riess, A. G., Strolger, L.-G., Casertano, S., et al. 2007, ApJ, 659, 98

Rigault, M. 2015, Presentation at the "Accurate Astrophysics. Correct Cosmology", London, UK, 16 July 2015 [UCL]

Rigault, M., Aldering, G., Kowalski, M., et al. 2015, ApJ, 802, 20 (R15)

Rigault, M., Copin, Y., Aldering, G., et al. 2013, A\&A, 560, A66 (R13)

Roman, M., Hardin, D., Betoule, M., et al. 2017, arXiv:1706.07697

Sako, M., Bassett, B., Becker, A. C., et al. 2014, arXiv:1401.3317

Salim, S., Rich, R. M., Charlot, S., et al. 2007, ApJS, 173, 267

Schmidt, B. P., Suntzeff, N. B., Phillips, M. M., et al. 1998, ApJ, 507,46

Smith, M., Nichol, R. C., Dilday, B., et al. 2012, ApJ, 755, 61

Stritzinger, M. D., Phillips, M. M., Boldt, L. N., et al. 2011, AJ, 142,156

Sullivan, M., Conley, A., Howell, D. A., et al. 2010, MNRAS, 406, 782

Sullivan, M., Ellis, R. S., Aldering, G., et al. 2003, MNRAS, 340, 1057

Sullivan, M., Le Borgne, D., Pritchet, C. J., et al. 2006, ApJ, 648,868

Taylor, J. 1997, Introduction to Error Analysis (2nd ed.: Mill Valley, CA: University Science Books)

Timmes, F. X., Brown, E. F., \& Truran, J. W. 2003, ApJL, 590, L83

Tremonti, C. A., Heckman, T. M., Kauffmann, G., et al. 2004, ApJ, 613, 898

Tripp, R. 1998, A\&A, 331, 815

Wood-Vasey, W. M., Miknaitis, G., Stubbs, C. W., et al. 2007, ApJ, 666, 694

Zhang, B. R., Childress, M. J., Davis, T. M., et al. 2017, MNRAS, 471, 2254 
$P(\operatorname{Ia\varepsilon })$
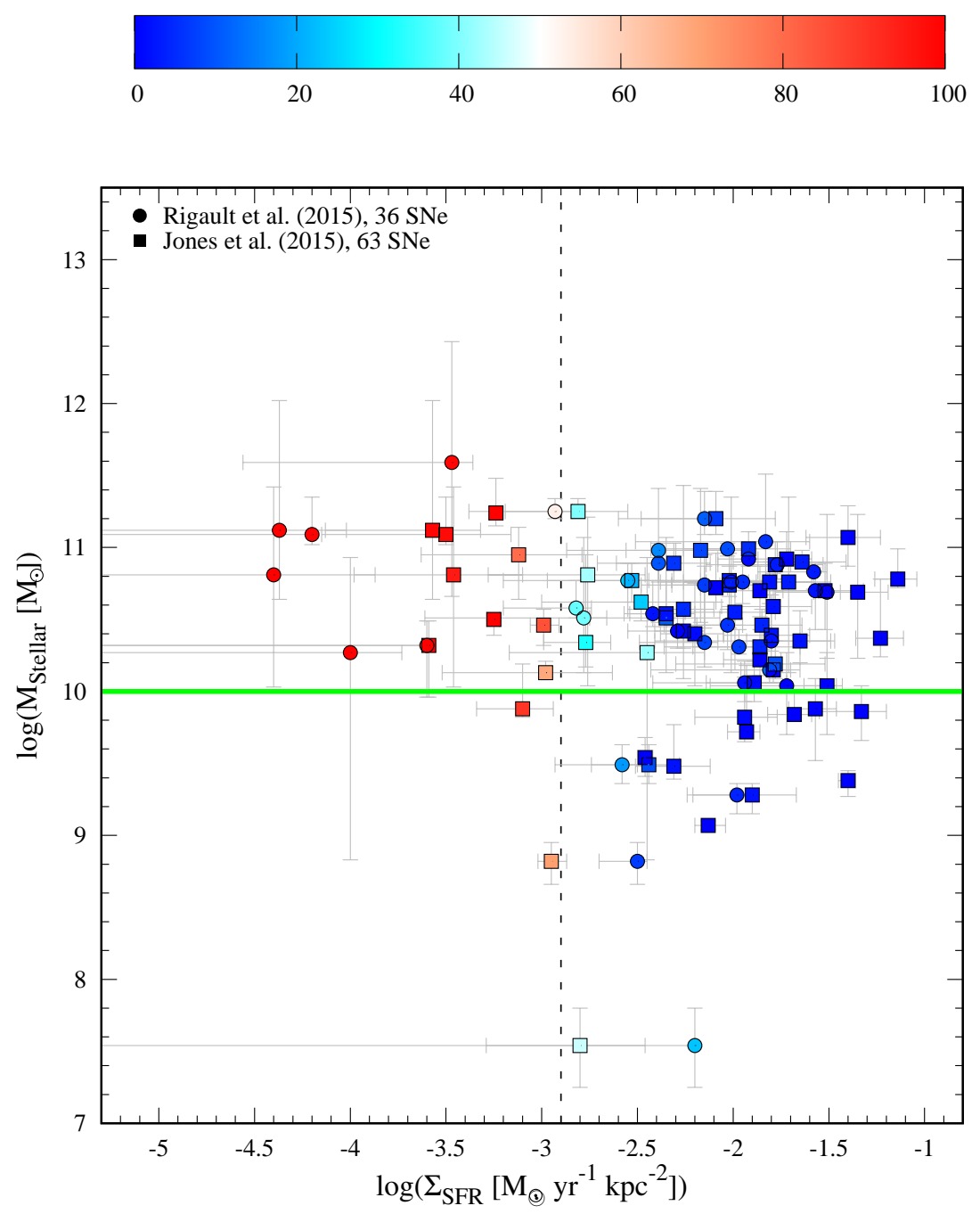

Figure 1. Our empirical method for globally star-forming host galaxies to select a SN sample without the local-global difference in star formation. The host galaxy local SFR surface density, $\log \left(\Sigma_{\mathrm{SFR}}\right)$, is plotted as a function of host galaxy stellar mass $\log \left(M_{\text {stellar }}\right)$, together with the probability of a locally passive environment $(\mathcal{P}(\mathrm{Ia} \epsilon))$. Host stellar masses are taken from Neill et al. (2009) and Kelly et al. (2010). The host local properties are drawn from R15 (filled circles) and J15 (filled squares), with $32 \mathrm{SNe}$ Ia in common. A clear distinction can be observed when we divide this figure into two regimes at $\log \left(M_{\text {stellar }}\right)=10$ (green horizontal line). For the high-mass hosts $\log \left(M_{\text {stellar }}\right)>10$, SNe Ia can arise either from locally passive (redder points) or locally star-forming environments (bluer points), while almost all of them occur only in locally star-forming environments for the low-mass hosts $\log \left(M_{\text {stellar }}\right) \leq 10$. Therefore, for the case of globally star-forming hosts, a sample without the local-global difference in star formation can be drawn from the low-mass hosts. SNe Ia are colored by $\mathcal{P}($ Ia $\epsilon$ ). The vertical dashed line shows the local star-formation surface density threshold, $\log \left(\Sigma_{\mathrm{SFR}}\right)=-2.9$ dex, taken from R15. 

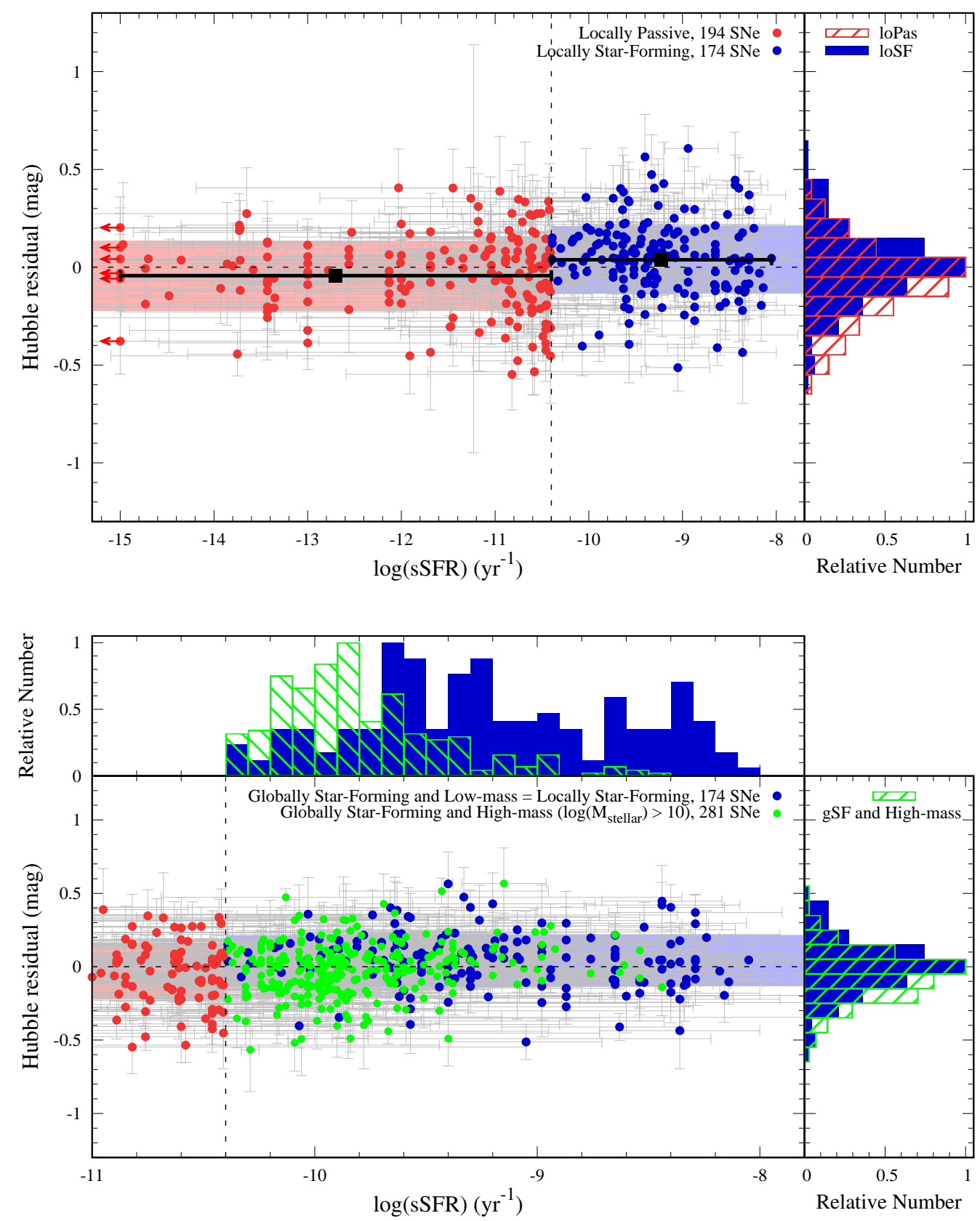

Figure 2. Upper panel: Environmental dependence of SN Ia luminosity from a sample without the local-global difference in star formation. The luminosity difference between SNe Ia in locally star-forming environments (blue circles) and those in locally passive environments (red circles) is $0.081 \pm 0.018 \mathrm{mag}(4.5 \sigma)$. This difference is consistent with the combined result of R15 $(0.094 \pm 0.025 \mathrm{mag})$, but is based on a sample 5 times larger and thus statistically more significant. Note that the r.m.s. scatter of SNe Ia in locally star-forming environments (blue shaded area) is $\sim 5 \%$ smaller than those in locally passive environments (red shaded area). The black squares represent the weighted-mean of Hubble residuals in bins of sSFR. The vertical dotted line indicates the limit distinguishing between passive and star-forming galaxies for our sample. Lower panel: The distribution of SNe Ia in globally star-forming and high-mass $\left(\log \left(M_{\text {stellar }}\right)>10\right)$ hosts (green circles), which have a sample with the localglobal difference in star formation. They have more negative Hubble residuals (right panel) and their hosts show less SFR than globally star-forming and low-mass (i.e., locally star-forming) hosts (upper panel). 


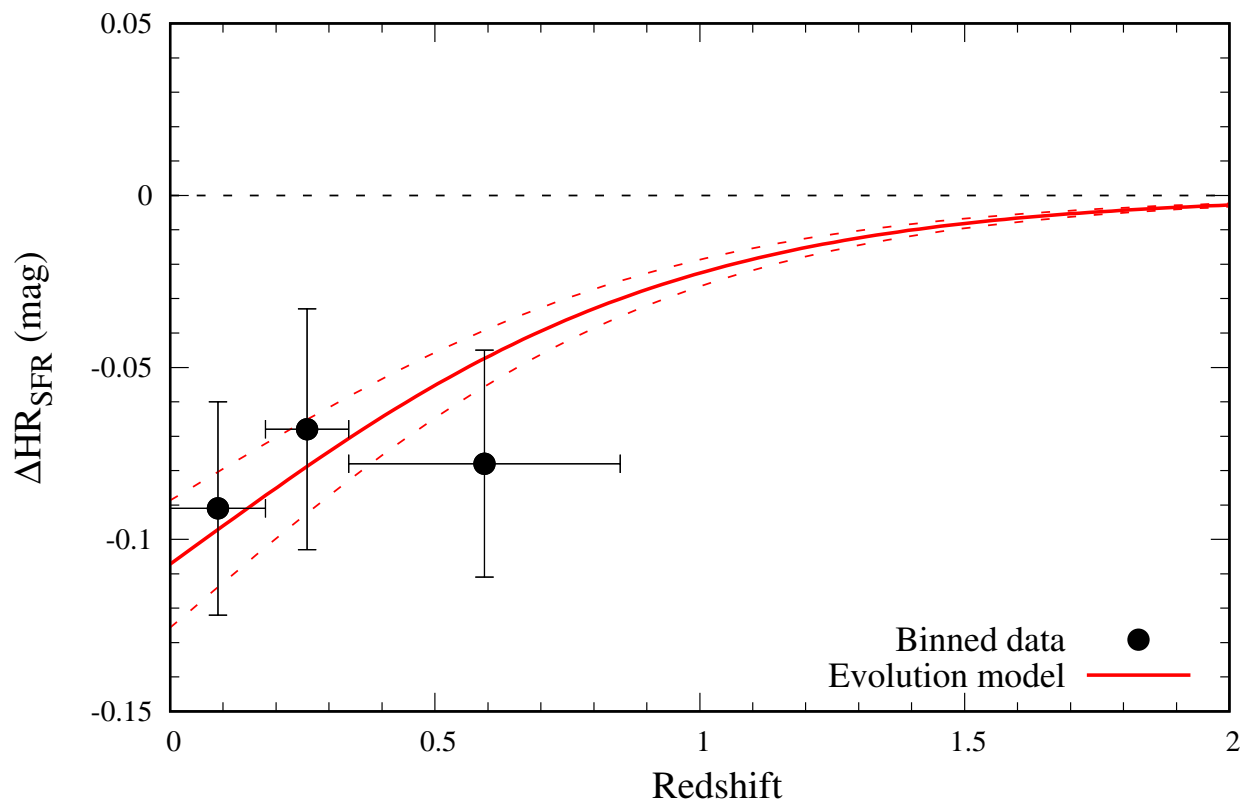

Figure 3. The observed and predicted evolution of the difference in Hubble residual between SNe Ia in locally passive and SNe Ia in locally star-forming environments $\left(\Delta \mathrm{HR}_{\mathrm{SFR}}\right)$. The filled circles are the observed $\Delta \mathrm{HR}_{\mathrm{SFR}}$ in three redshift bins from this study. The red solid line shows the simple evolution model from Eq. (4). This model is consistent with our observed data, but further SN Ia data are required to test the model in detail. The red dotted lines show $\pm 1 \sigma$ ranges of the evolution model. 
Table 1. The sample sizes for each group of host galaxies.

\begin{tabular}{cccc}
\hline \hline \multirow{2}{*}{ YONSEI Cosmology } & \multicolumn{3}{c}{ Host groups } \\
\cline { 2 - 4 } & Mass & Global sSFR & Local group from this work \\
\hline 941 & $648(657)$ & $649(657)$ & $368(373)$ \\
\hline
\end{tabular}

Note-The number in parenthesis is the value before applying Chauvenet's criterion (see Section 2). 
Table 2. YONSEI Supernova Catalog and host 'global' properties

\begin{tabular}{|c|c|c|c|c|c|c|c|c|c|c|}
\hline \multirow[b]{2}{*}{ Name } & \multirow[b]{2}{*}{ Survey } & \multirow[b]{2}{*}{$\mathrm{z}_{C M B}$} & \multicolumn{2}{|c|}{ SALT2 } & \multicolumn{3}{|c|}{ Host Mass } & \multicolumn{3}{|c|}{ Global sSFR } \\
\hline & & & $\begin{array}{c}\text { HR } \\
(\mathrm{mag})\end{array}$ & $\sigma_{H R}$ & $\begin{array}{c}\log \left(\mathrm{M}_{\text {stellar }}\right) \\
\left(\mathrm{M}_{\odot}\right)\end{array}$ & $-\delta$ & $+\delta$ & $\begin{array}{c}\log (\mathrm{sSFR}) \\
\left(\mathrm{yr}^{-1}\right)\end{array}$ & $-\delta$ & $+\delta$ \\
\hline 1991ag & LOWZ & 0.014 & -0.088 & 0.122 & 9.07 & 0.03 & 0.03 & -8.66 & 0.06 & 0.08 \\
\hline $1992 \mathrm{P}$ & LOWZ & 0.026 & 0.134 & 0.161 & 10.34 & 0.10 & 0.14 & -9.98 & 0.88 & 0.63 \\
\hline $1992 \mathrm{ag}$ & LOWZ & 0.026 & -0.714 & 0.156 & 10.02 & 0.10 & 1.04 & -9.88 & 1.23 & 0.93 \\
\hline $1992 \mathrm{bc}$ & LOWZ & 0.020 & 0.063 & 0.101 & 9.72 & 0.53 & 0.70 & -9.62 & 0.94 & 1.12 \\
\hline $1992 \mathrm{bl}$ & LOWZ & 0.043 & -0.069 & 0.168 & 11.81 & 0.65 & 0.46 & -10.85 & 0.71 & 1.58 \\
\hline 722 & SDSS3 & 0.085 & 0.055 & 0.115 & 10.93 & 0.01 & 0.01 & -12.56 & 0.70 & 0.70 \\
\hline 739 & SDSS3 & 0.106 & -0.117 & 0.151 & 11.17 & 0.03 & 0.03 & -10.85 & 0.17 & 0.17 \\
\hline 744 & SDSS3 & 0.127 & -0.061 & 0.133 & 10.62 & 0.11 & 0.11 & -10.24 & 0.22 & 0.22 \\
\hline 762 & SDSS3 & 0.190 & 0.175 & 0.120 & 11.23 & 0.01 & 0.01 & -10.39 & 0.40 & 0.40 \\
\hline 774 & SDSS3 & 0.092 & -0.007 & 0.101 & 10.81 & 0.02 & 0.02 & -10.31 & 0.10 & 0.10 \\
\hline 03D1ar & SNLS3 & 0.408 & 0.066 & 0.135 & 10.46 & 0.18 & 0.18 & -8.98 & 0.39 & 0.39 \\
\hline 03D1au & SNLS3 & 0.504 & -0.061 & 0.115 & 9.75 & 0.04 & 0.04 & -9.63 & 0.13 & 0.13 \\
\hline 03D1aw & SNLS3 & 0.582 & 0.177 & 0.141 & 9.57 & 0.17 & 0.17 & -9.19 & 0.26 & 0.26 \\
\hline 03D1ax & SNLS3 & 0.496 & -0.016 & 0.110 & 11.70 & 0.08 & 0.08 & -12.78 & 0.68 & 0.68 \\
\hline 03D1bp & SNLS3 & 0.347 & 0.133 & 0.103 & 11.03 & 0.02 & 0.02 & -10.20 & 0.06 & 0.06 \\
\hline
\end{tabular}

NoтE-Table 2 is published in its entirety in the machine-readable format. A portion is shown here for guidance regarding its form and content. 
Table 3. Host stellar mass and 'local' properties for the Low- $z$ sample

\begin{tabular}{|c|c|c|c|c|c|c|c|c|c|c|c|c|c|}
\hline \multirow[b]{2}{*}{ Name } & \multirow[b]{2}{*}{$\mathrm{z}_{C M B}$} & \multicolumn{3}{|c|}{ Host Mass } & \multirow{2}{*}{$\begin{array}{c}\text { Global } \\
\text { Host Class }\end{array}$} & \multicolumn{4}{|c|}{ R15 Local SFR } & \multicolumn{4}{|c|}{ J15 Local SFR } \\
\hline & & $\begin{array}{c}\log \left(\mathrm{M}_{\text {stellar }}\right) \\
\left(\mathrm{M}_{\odot}\right)\end{array}$ & $-\delta$ & $+\delta$ & & $\begin{array}{c}\log \left(\Sigma_{S F R}\right) \\
\left(\mathrm{M}_{\odot} \mathrm{yr}^{-1} \mathrm{kpc}^{-2}\right)\end{array}$ & $-\delta$ & $+\delta$ & $\begin{array}{c}P(I a \epsilon) \\
(\%)\end{array}$ & $\begin{array}{c}\log \left(\Sigma_{S F R}\right) \\
\left(\mathrm{M}_{\odot} \mathrm{yr}^{-1} \mathrm{kpc}^{-2}\right)\end{array}$ & $-\delta$ & $+\delta$ & $\begin{array}{c}P(I a \epsilon) \\
(\%)\end{array}$ \\
\hline $1991 U$ & 0.033 & 11.04 & 0.70 & 0.47 & SF & -1.83 & 0.68 & 0.23 & 6 & $\ldots$ & $\ldots$ & $\cdots$ & $\cdots$ \\
\hline 1991ag & 0.014 & 9.07 & 0.03 & 0.03 & SF & $\cdots$ & $\cdots$ & $\ldots$ & $\ldots$ & -2.13 & 0.07 & 0.09 & 0 \\
\hline $1992 \mathrm{bl}$ & 0.043 & 11.81 & 0.65 & 0.46 & $\mathrm{~Pa}$ & -3.48 & 99.00 & 0.43 & 96 & -3.05 & 0.46 & 0.23 & 80 \\
\hline 1992bo & 0.018 & 12.13 & 1.20 & 0.14 & $\mathrm{~Pa}$ & $\cdots$ & $\cdots$ & $\cdots$ & $\cdots$ & -4.28 & 99.00 & 0.00 & 100 \\
\hline $1993 \mathrm{H}$ & 0.025 & 10.51 & 0.38 & 0.56 & SF & -2.78 & 0.83 & 0.12 & 39 & -2.35 & 0.40 & 0.28 & 11 \\
\hline $1993 \mathrm{ac}$ & 0.049 & 11.44 & 0.03 & 0.04 & $\mathrm{~Pa}$ & -4.24 & 99.00 & 0.30 & 98 & -2.73 & 0.61 & 0.32 & 51 \\
\hline 1993ae & 0.018 & 10.35 & 0.06 & 1.55 & $\mathrm{~Pa}$ & $\cdots$ & $\cdots$ & $\cdots$ & $\cdots$ & -4.54 & 99.00 & 0.00 & 100 \\
\hline $1994 \mathrm{M}$ & 0.024 & 11.04 & 0.11 & 0.19 & $\mathrm{~Pa}$ & -4.30 & 99.00 & 0.00 & 100 & -3.34 & 99.00 & 0.00 & 100 \\
\hline $1994 \mathrm{Q}$ & 0.029 & 9.84 & 0.04 & 0.16 & SF & $\ldots$ & $\ldots$ & $\ldots$ & $\ldots$ & -1.68 & 0.14 & 0.09 & 0 \\
\hline $1994 \mathrm{~S}$ & 0.016 & 10.50 & 0.11 & 0.03 & SF & $\ldots$ & $\ldots$ & $\ldots$ & $\ldots$ & -3.25 & 0.04 & 0.03 & 100 \\
\hline $1994 \mathrm{~T}$ & 0.036 & 9.60 & 0.37 & 1.00 & $\mathrm{~Pa}$ & -4.30 & 99.00 & 0.00 & 100 & -3.47 & 0.69 & 0.28 & 98 \\
\hline 1996bo & 0.016 & 10.37 & 0.13 & 0.41 & SF & $\ldots$ & $\ldots$ & $\ldots$ & $\ldots$ & -1.23 & 0.13 & 0.12 & 0 \\
\hline $1997 \mathrm{cn}$ & 0.017 & 11.42 & 0.04 & 0.04 & $\mathrm{~Pa}$ & $\ldots$ & $\ldots$ & $\ldots$ & $\ldots$ & -3.29 & 0.17 & 0.18 & 99 \\
\hline $1998 \mathrm{ab}$ & 0.028 & 10.59 & 0.04 & 0.23 & SF & $\ldots$ & $\ldots$ & $\ldots$ & $\ldots$ & -1.79 & 0.27 & 0.20 & 2 \\
\hline 1998de & 0.016 & 11.25 & 0.38 & 0.65 & $\mathrm{~Pa}$ & $\ldots$ & $\ldots$ & $\ldots$ & $\cdots$ & -3.41 & 99.00 & 0.00 & 100 \\
\hline $1998 \mathrm{dx}$ & 0.054 & 11.72 & 0.55 & 0.87 & $\mathrm{~Pa}$ & -3.70 & 99.00 & 0.00 & 97 & -2.77 & 99.00 & 0.00 & 93 \\
\hline $1998 \mathrm{ec}$ & 0.020 & 10.57 & 0.48 & 0.86 & SF & $\cdots$ & $\cdots$ & $\ldots$ & $\cdots$ & -2.26 & 0.29 & 0.16 & 5 \\
\hline 1999X & 0.026 & 10.13 & 0.03 & 0.08 & $\mathrm{SF}$ & $\ldots$ & $\ldots$ & $\cdots$ & $\ldots$ & -2.98 & 0.54 & 0.35 & 68 \\
\hline 1999aa & 0.015 & 10.72 & 0.10 & 0.24 & SF & $\ldots$ & $\ldots$ & $\ldots$ & $\ldots$ & -2.09 & 0.08 & 0.06 & 0 \\
\hline $1999 \mathrm{cc}$ & 0.032 & 10.99 & 0.05 & 0.04 & SF & -2.03 & 0.40 & 0.09 & 9 & -1.92 & 0.34 & 0.22 & 5 \\
\hline $1999 \mathrm{cp}$ & 0.010 & 9.48 & 0.09 & 0.29 & SF & $\ldots$ & $\ldots$ & $\ldots$ & $\ldots$ & -2.31 & 0.20 & 0.19 & 2 \\
\hline $1999 \mathrm{da}$ & 0.013 & 10.91 & 0.12 & 0.22 & $\mathrm{~Pa}$ & $\ldots$ & $\ldots$ & $\ldots$ & $\cdots$ & -4.02 & 0.53 & 0.32 & 100 \\
\hline $1999 \mathrm{dq}$ & 0.014 & 10.78 & 0.06 & 0.21 & SF & $\ldots$ & $\ldots$ & $\ldots$ & $\ldots$ & -1.14 & 0.12 & 0.10 & 0 \\
\hline 2000ca & 0.025 & 10.04 & 0.34 & 0.23 & SF & -1.72 & 0.40 & 0.26 & 0 & -1.51 & 0.12 & 0.08 & 0 \\
\hline $2000 \mathrm{dk}$ & 0.016 & 11.54 & 1.37 & 0.02 & $\mathrm{~Pa}$ & $\ldots$ & $\ldots$ & $\ldots$ & $\cdots$ & -3.75 & 0.07 & 0.06 & 100 \\
\hline $2000 \mathrm{fa}$ & 0.022 & 9.82 & 0.17 & 0.28 & $\mathrm{SF}$ & $\ldots$ & $\ldots$ & $\ldots$ & $\ldots$ & -1.94 & 0.26 & 0.17 & 1 \\
\hline $2001 \mathrm{~N}$ & 0.022 & 10.77 & 0.11 & 0.19 & SF & $\ldots$ & $\ldots$ & $\ldots$ & $\ldots$ & -2.02 & 0.30 & 0.21 & 3 \\
\hline 2001ay & 0.031 & 10.62 & 0.13 & 0.15 & $\mathrm{SF}$ & $\ldots$ & $\cdots$ & $\cdots$ & $\ldots$ & -2.48 & 0.52 & 0.39 & 24 \\
\hline $2001 \mathrm{ba}$ & 0.031 & 10.98 & 0.50 & 0.43 & SF & -2.39 & 0.48 & 0.09 & 16 & -2.17 & 0.39 & 0.28 & 8 \\
\hline 2001en & 0.016 & 10.38 & 0.15 & 0.15 & $\mathrm{~Pa}$ & $\ldots$ & $\ldots$ & $\ldots$ & $\ldots$ & -2.30 & 0.30 & 0.23 & 8 \\
\hline $2001 \mathrm{fe}$ & 0.014 & 10.22 & 0.11 & 0.12 & SF & $\ldots$ & $\ldots$ & $\ldots$ & $\ldots$ & -1.86 & 0.19 & 0.11 & 0 \\
\hline $2001 \mathrm{gb}$ & 0.027 & 10.39 & 0.11 & 0.28 & SF & $\ldots$ & $\ldots$ & $\ldots$ & $\cdots$ & -1.80 & 0.25 & 0.20 & 2 \\
\hline $2001 \mathrm{ic}$ & 0.043 & 11.70 & 0.06 & 0.04 & $\mathrm{~Pa}$ & -5.80 & 99.00 & 0.00 & 100 & -2.30 & 99.00 & 0.00 & 77 \\
\hline 2001ie & 0.031 & 10.99 & 0.07 & 0.21 & $\mathrm{~Pa}$ & -3.90 & 99.00 & 0.00 & 100 & -3.68 & 0.65 & 0.35 & 99 \\
\hline $2002 \mathrm{G}$ & 0.035 & 10.77 & 0.15 & 0.12 & SF & -2.55 & 0.65 & 0.11 & 24 & -2.53 & 0.44 & 0.36 & 21 \\
\hline $2002 \mathrm{bf}$ & 0.025 & 10.62 & 0.07 & 0.12 & $\mathrm{~Pa}$ & -2.18 & 0.48 & 0.10 & 12 & -2.23 & 0.37 & 0.22 & 10 \\
\hline 2002 de & 0.028 & 10.83 & 0.12 & 0.03 & SF & -1.58 & 0.29 & 0.07 & 3 & $\cdots$ & $\cdots$ & $\cdots$ & $\cdots$ \\
\hline $2002 \mathrm{dp}$ & 0.011 & 10.40 & 0.36 & 0.35 & SF & $\ldots$ & $\ldots$ & $\ldots$ & $\ldots$ & -2.20 & 0.18 & 0.15 & 1 \\
\hline 2002ha & 0.013 & 11.09 & 0.13 & 0.14 & $\mathrm{~Pa}$ & $\ldots$ & $\ldots$ & $\ldots$ & $\ldots$ & -2.53 & 0.21 & 0.14 & 6 \\
\hline 2002he & 0.025 & 11.12 & 0.48 & 0.90 & SF & -4.37 & 99.00 & 0.35 & 100 & -3.57 & 0.56 & 0.25 & 99 \\
\hline $2002 \mathrm{hu}$ & 0.038 & 10.27 & 1.44 & 0.66 & SF & -4.00 & 99.00 & 0.27 & 100 & -2.45 & 0.72 & 0.30 & 42 \\
\hline $2002 \mathrm{hw}$ & 0.016 & 10.38 & 0.14 & 0.18 & $\mathrm{~Pa}$ & $\ldots$ & $\ldots$ & $\ldots$ & $\ldots$ & -2.17 & 0.33 & 0.23 & 6 \\
\hline 2002jy & 0.019 & 10.46 & 0.14 & 0.11 & SF & $\cdots$ & $\ldots$ & $\cdots$ & $\ldots$ & -2.99 & 0.07 & 0.08 & 85 \\
\hline $2003 \mathrm{U}$ & 0.028 & 10.74 & 0.30 & 0.13 & SF & -2.15 & 0.33 & 0.08 & 7 & -2.02 & 0.30 & 0.19 & 4 \\
\hline $2003 \mathrm{~W}$ & 0.021 & 10.55 & 0.40 & 0.25 & $\mathrm{SF}$ & $\cdots$ & $\cdots$ & $\cdots$ & $\cdots$ & -1.99 & 0.26 & 0.21 & 3 \\
\hline $2003 \mathrm{cq}$ & 0.034 & 11.20 & 0.11 & 0.19 & SF & -2.15 & 0.45 & 0.09 & 11 & -2.09 & 0.39 & 0.31 & 7 \\
\hline $2003 \mathrm{fa}$ & 0.039 & 10.81 & 0.78 & 0.61 & SF & -4.40 & 99.00 & 0.53 & 100 & -3.46 & 0.52 & 0.36 & 95 \\
\hline
\end{tabular}


Table 3 (continued)

\begin{tabular}{|c|c|c|c|c|c|c|c|c|c|c|c|c|c|}
\hline \multirow[b]{2}{*}{ Name } & \multirow[b]{2}{*}{$\mathrm{z}_{C M B}$} & \multicolumn{3}{|c|}{ Host Mass } & \multirow{2}{*}{$\begin{array}{c}\text { Global } \\
\text { Host Class }\end{array}$} & \multicolumn{4}{|c|}{ R15 Local SFR } & \multicolumn{4}{|c|}{ J15 Local SFR } \\
\hline & & $\begin{array}{c}\log \left(\mathrm{M}_{\text {stellar }}\right) \\
\left(\mathrm{M}_{\odot}\right)\end{array}$ & $-\delta$ & $+\delta$ & & $\begin{array}{c}\log \left(\Sigma_{S F R}\right) \\
\left(\mathrm{M}_{\odot} \mathrm{yr}^{-1} \mathrm{kpc}^{-2}\right)\end{array}$ & $-\delta$ & $+\delta$ & $\begin{array}{c}P(I a \epsilon) \\
(\%)\end{array}$ & $\begin{array}{c}\log \left(\Sigma_{S F R}\right) \\
\left(\mathrm{M}_{\odot} \mathrm{yr}^{-1} \mathrm{kpc}^{-2}\right)\end{array}$ & $-\delta$ & $+\delta$ & $\begin{array}{c}P(\text { Ia }) \\
(\%)\end{array}$ \\
\hline $2003 \mathrm{hu}$ & 0.075 & 10.90 & 0.04 & 0.08 & SF & $\cdots$ & $\cdots$ & $\cdots$ & $\cdots$ & -1.64 & 0.36 & 0.23 & 3 \\
\hline 2003 ic & 0.054 & 11.70 & 0.03 & 0.27 & $\mathrm{~Pa}$ & -3.56 & 0.18 & 0.05 & 100 & -3.37 & 0.19 & 0.08 & 100 \\
\hline $2004 \mathrm{~L}$ & 0.033 & 10.35 & 0.15 & 0.21 & SF & -1.80 & 0.61 & 0.31 & 2 & -1.65 & 0.28 & 0.18 & 2 \\
\hline 2004as & 0.032 & 9.28 & 0.13 & 0.08 & SF & -1.98 & 0.26 & 0.07 & 4 & -1.90 & 0.31 & 0.23 & 2 \\
\hline 2005 eq & 0.028 & 10.58 & 0.07 & 0.19 & SF & -2.82 & 0.38 & 0.08 & 38 & $\cdots$ & $\cdots$ & $\cdots$ & $\cdots$ \\
\hline $2005 \mathrm{hc}$ & 0.045 & 10.54 & 0.09 & 0.23 & SF & -2.42 & 0.15 & 0.04 & 2 & -2.35 & 0.19 & 0.15 & 2 \\
\hline $2005 \mathrm{hj}$ & 0.057 & 9.49 & 0.13 & 0.14 & $\mathrm{SF}$ & -2.58 & 0.35 & 0.08 & 18 & -2.44 & 0.30 & 0.16 & 9 \\
\hline $2005 \mathrm{hk}$ & 0.012 & 9.54 & 0.13 & 0.14 & $\mathrm{SF}$ & $\cdots$ & $\cdots$ & $\cdots$ & $\cdots$ & -2.46 & 0.04 & 0.04 & 0 \\
\hline $2005 \mathrm{iq}$ & 0.033 & 10.34 & 0.17 & 0.61 & SF & -2.15 & 0.34 & 0.07 & 8 & -2.77 & 0.22 & 0.13 & 32 \\
\hline 2005ir & 0.075 & 10.15 & 0.09 & 0.05 & SF & -1.81 & 0.62 & 0.11 & 12 & -1.79 & 0.17 & 0.14 & 0 \\
\hline $2005 \mathrm{kc}$ & 0.014 & 10.97 & 0.13 & 0.12 & $\mathrm{~Pa}$ & $\cdots$ & $\ldots$ & $\ldots$ & $\cdots$ & -1.76 & 0.22 & 0.17 & 0 \\
\hline $2005 \mathrm{ls}$ & 0.021 & 9.86 & 0.20 & 0.18 & $\mathrm{SF}$ & $\cdots$ & $\ldots$ & $\ldots$ & $\cdots$ & -1.33 & 0.22 & 0.13 & 0 \\
\hline $2005 \mathrm{mc}$ & 0.026 & 10.95 & 0.09 & 0.11 & $\mathrm{~Pa}$ & -2.54 & 0.31 & 0.07 & 14 & -3.68 & 0.16 & 0.11 & 100 \\
\hline $2005 \mathrm{~ms}$ & 0.026 & 10.32 & 0.36 & 0.17 & SF & -3.60 & 99.00 & 0.00 & 98 & -3.59 & 99.00 & 0.00 & 100 \\
\hline $2005 \mathrm{mz}$ & 0.017 & 11.24 & 0.09 & 0.24 & SF & $\ldots$ & $\ldots$ & $\ldots$ & $\ldots$ & -3.24 & 0.03 & 0.04 & 100 \\
\hline $2006 \mathrm{~N}$ & 0.014 & 10.82 & 0.23 & 0.06 & $\mathrm{~Pa}$ & $\ldots$ & $\ldots$ & $\ldots$ & $\ldots$ & -3.58 & 0.14 & 0.12 & 100 \\
\hline $2006 \mathrm{~S}$ & 0.033 & 10.46 & 0.17 & 0.11 & SF & -2.03 & 0.33 & 0.08 & 6 & -1.85 & 0.34 & 0.21 & 4 \\
\hline $2006 \mathrm{ac}$ & 0.024 & 10.92 & 0.05 & 0.19 & $\mathrm{SF}$ & -1.92 & 0.16 & 0.05 & 1 & -1.72 & 0.23 & 0.13 & 0 \\
\hline 2006al & 0.069 & 10.26 & 0.03 & 0.29 & $\mathrm{~Pa}$ & -4.00 & 99.00 & 0.13 & 100 & -3.74 & 0.42 & 0.29 & 100 \\
\hline 2006an & 0.065 & 7.54 & 0.29 & 0.26 & SF & -2.20 & 99.00 & 0.00 & 23 & -2.80 & 0.49 & 0.34 & 46 \\
\hline 2006ar & 0.023 & 9.72 & 0.05 & 0.46 & SF & $\ldots$ & $\ldots$ & $\ldots$ & $\cdots$ & -1.93 & 0.10 & 0.07 & 0 \\
\hline $2006 a x$ & 0.018 & 10.81 & 0.77 & 0.40 & $\mathrm{SF}$ & $\ldots$ & $\ldots$ & $\ldots$ & $\cdots$ & -2.76 & 0.52 & 0.30 & 43 \\
\hline $2006 \mathrm{az}$ & 0.032 & 11.28 & 0.02 & 0.26 & $\mathrm{~Pa}$ & -3.43 & 0.39 & 0.07 & 100 & -3.20 & 0.21 & 0.19 & 96 \\
\hline $2006 \mathrm{bd}$ & 0.026 & 11.09 & 0.28 & 0.09 & $\mathrm{~Pa}$ & -4.50 & 99.00 & 0.12 & 100 & -4.45 & 0.52 & 0.30 & 100 \\
\hline $2006 \mathrm{bt}$ & 0.033 & 11.09 & 0.07 & 0.26 & SF & -4.20 & 99.00 & 0.00 & 100 & -3.50 & 0.65 & 0.34 & 98 \\
\hline $2006 \mathrm{bw}$ & 0.031 & 10.03 & 0.08 & 0.24 & $\mathrm{~Pa}$ & -4.90 & 99.00 & 0.00 & 100 & -3.90 & 99.00 & 0.00 & 100 \\
\hline $2006 \mathrm{bz}$ & 0.028 & 10.42 & 0.29 & 0.09 & $\mathrm{~Pa}$ & -4.45 & 0.27 & 0.10 & 100 & -4.41 & 0.13 & 0.09 & 100 \\
\hline $2006 \mathrm{cf}$ & 0.042 & 10.88 & 0.10 & 0.16 & $\mathrm{SF}$ & -1.77 & 0.31 & 0.07 & 5 & -1.78 & 0.36 & 0.25 & 3 \\
\hline $2006 \mathrm{cg}$ & 0.029 & 9.92 & 0.12 & 0.27 & $\mathrm{~Pa}$ & -4.22 & 0.41 & 0.07 & 100 & -4.13 & 0.28 & 0.20 & 100 \\
\hline $2006 \mathrm{cj}$ & 0.068 & 10.42 & 0.12 & 0.20 & SF & -2.29 & 0.26 & 0.10 & 0 & -2.26 & 0.15 & 0.11 & 0 \\
\hline $2006 \mathrm{cp}$ & 0.023 & 9.88 & 0.06 & 0.31 & SF & $\ldots$ & $\ldots$ & $\ldots$ & $\cdots$ & -3.10 & 0.24 & 0.16 & 90 \\
\hline $2006 \mathrm{cq}$ & 0.049 & 10.89 & 0.13 & 0.14 & SF & -2.39 & 0.32 & 0.08 & 10 & -2.31 & 0.30 & 0.19 & 6 \\
\hline $2006 \mathrm{cs}$ & 0.024 & 11.09 & 0.34 & 0.05 & $\mathrm{~Pa}$ & -3.79 & 0.70 & 0.13 & 100 & -3.46 & 0.42 & 0.23 & 99 \\
\hline $2006 \mathrm{ef}$ & 0.017 & 10.70 & 0.04 & 0.08 & $\mathrm{~Pa}$ & $\ldots$ & $\ldots$ & $\ldots$ & $\ldots$ & -3.84 & 0.10 & 0.07 & 100 \\
\hline 2006ej & 0.019 & 11.00 & 0.24 & 0.02 & $\mathrm{~Pa}$ & $\cdots$ & $\ldots$ & $\cdots$ & $\cdots$ & -3.56 & 0.29 & 0.21 & 100 \\
\hline 2006en & 0.031 & 10.69 & 0.46 & 0.54 & SF & -1.51 & 0.27 & 0.07 & 2 & -1.35 & 0.23 & 0.16 & 0 \\
\hline $2006 \mathrm{hb}$ & 0.015 & 10.95 & 0.31 & 0.19 & SF & $\ldots$ & $\ldots$ & $\ldots$ & $\ldots$ & -3.12 & 0.51 & 0.33 & 80 \\
\hline $2006 \mathrm{kf}$ & 0.021 & 10.97 & 0.24 & 0.09 & $\mathrm{~Pa}$ & $\ldots$ & $\ldots$ & $\ldots$ & $\cdots$ & -3.12 & 0.50 & 0.24 & 89 \\
\hline 2006le & 0.017 & 10.19 & 0.10 & 0.16 & SF & $\ldots$ & $\ldots$ & $\ldots$ & $\ldots$ & -1.78 & 0.52 & 0.26 & 9 \\
\hline $2006 \mathrm{mo}$ & 0.036 & 10.82 & 0.02 & 0.17 & $\mathrm{~Pa}$ & -3.81 & 0.27 & 0.04 & 100 & -3.66 & 0.24 & 0.16 & 100 \\
\hline $2006 \mathrm{nz}$ & 0.037 & 10.62 & 0.14 & 0.04 & $\mathrm{~Pa}$ & -3.77 & 0.17 & 0.04 & 100 & -3.55 & 0.11 & 0.07 & 100 \\
\hline 2006oa & 0.058 & 8.82 & 0.16 & 0.13 & SF & -2.50 & 0.20 & 0.05 & 7 & -2.95 & 0.07 & 0.08 & 70 \\
\hline 2006ob & 0.058 & 11.25 & 0.05 & 0.09 & SF & -2.93 & 0.45 & 0.09 & 53 & -2.81 & 0.38 & 0.26 & 40 \\
\hline 2006on & 0.068 & 10.30 & 0.05 & 0.08 & $\mathrm{~Pa}$ & -4.70 & 99.00 & 0.00 & 100 & -3.27 & 99.00 & 0.00 & 100 \\
\hline 2006or & 0.022 & 11.07 & 0.20 & 0.22 & $\mathrm{SF}$ & $\ldots$ & $\ldots$ & $\ldots$ & $\ldots$ & -1.40 & 0.22 & 0.17 & 0 \\
\hline 2006 os & 0.032 & 11.59 & 0.93 & 0.84 & SF & -3.47 & 1.09 & 0.11 & 99 & $\ldots$ & $\cdots$ & $\cdots$ & $\cdots$ \\
\hline $2006 \mathrm{sr}$ & 0.023 & 10.70 & 0.18 & 0.17 & SF & $\ldots$ & $\ldots$ & $\ldots$ & $\ldots$ & -1.86 & 0.13 & 0.12 & 0 \\
\hline 2006te & 0.032 & 10.31 & 0.05 & 0.12 & $\mathrm{SF}$ & -1.97 & 0.39 & 0.08 & 7 & -1.86 & 0.35 & 0.21 & 4 \\
\hline $2007 \mathrm{~F}$ & 0.024 & 10.06 & 0.13 & 0.15 & SF & -1.94 & 0.48 & 0.22 & 2 & -1.89 & 0.25 & 0.17 & 1 \\
\hline
\end{tabular}


Table 3 (continued)

\begin{tabular}{|c|c|c|c|c|c|c|c|c|c|c|c|c|c|}
\hline \multirow[b]{2}{*}{ Name } & \multirow[b]{2}{*}{$\mathrm{z}_{C M B}$} & \multicolumn{3}{|c|}{ Host Mass } & \multirow{2}{*}{$\begin{array}{c}\text { Global } \\
\text { Host Class }\end{array}$} & \multicolumn{4}{|c|}{ R15 Local SFR } & \multicolumn{4}{|c|}{ J15 Local SFR } \\
\hline & & $\begin{array}{c}\log \left(\mathrm{M}_{\text {stellar }}\right) \\
\left(\mathrm{M}_{\odot}\right)\end{array}$ & $-\delta$ & $+\delta$ & & $\begin{array}{c}\log \left(\Sigma_{S F R}\right) \\
\left(\mathrm{M}_{\odot} \mathrm{yr}^{-1} \mathrm{kpc}^{-2}\right)\end{array}$ & $-\delta$ & $+\delta$ & $\begin{array}{c}P(I a \epsilon) \\
(\%)\end{array}$ & $\begin{array}{c}\log \left(\Sigma_{S F R}\right) \\
\left(\mathrm{M}_{\odot} \mathrm{yr}^{-1} \mathrm{kpc}^{-2}\right)\end{array}$ & $-\delta$ & $+\delta$ & $\begin{array}{l}P(I a \epsilon) \\
(\%)\end{array}$ \\
\hline $2007 \mathrm{O}$ & 0.036 & 10.70 & 0.27 & 0.19 & $\mathrm{SF}$ & -1.57 & 0.24 & 0.06 & 2 & -1.52 & 0.26 & 0.20 & 0 \\
\hline $2007 \mathrm{R}$ & 0.031 & 10.98 & 0.13 & 0.14 & $\mathrm{~Pa}$ & -1.66 & 0.28 & 0.07 & 3 & -1.52 & 0.27 & 0.20 & 2 \\
\hline $2007 \mathrm{~S}$ & 0.015 & 9.88 & 0.36 & 0.21 & $\mathrm{SF}$ & $\cdots$ & $\cdots$ & $\cdots$ & $\cdots$ & -1.57 & 0.14 & 0.11 & 0 \\
\hline 2007ae & 0.064 & 11.44 & 0.19 & 0.17 & $\mathrm{~Pa}$ & -2.88 & 0.44 & 0.07 & 47 & -2.32 & 0.60 & 0.38 & 22 \\
\hline 2007ar & 0.053 & 11.51 & 0.04 & 0.04 & $\mathrm{~Pa}$ & $\cdots$ & $\cdots$ & $\cdots$ & $\cdots$ & -3.58 & 0.66 & 0.32 & 99 \\
\hline $2007 \mathrm{au}$ & 0.020 & 12.24 & 0.96 & 0.10 & $\mathrm{~Pa}$ & $\cdots$ & $\cdots$ & $\cdots$ & $\cdots$ & -4.47 & 0.53 & 0.29 & 100 \\
\hline $2007 \mathrm{ba}$ & 0.039 & 11.05 & 0.10 & 0.18 & $\mathrm{~Pa}$ & -3.64 & 0.11 & 0.03 & 100 & -3.48 & 0.12 & 0.08 & 100 \\
\hline $2007 \mathrm{bd}$ & 0.032 & 10.76 & 0.15 & 0.14 & $\mathrm{SF}$ & -1.95 & 0.31 & 0.07 & 5 & -1.81 & 0.33 & 0.22 & 2 \\
\hline $2007 \mathrm{bz}$ & 0.023 & 9.38 & 0.11 & 0.07 & $\mathrm{SF}$ & $\cdots$ & $\cdots$ & $\ldots$ & $\cdots$ & -1.40 & 0.05 & 0.04 & 0 \\
\hline $2007 \mathrm{cg}$ & 0.034 & 10.76 & 0.63 & 0.59 & $\mathrm{SF}$ & -2.01 & 0.37 & 0.09 & 8 & -1.71 & 0.33 & 0.22 & 3 \\
\hline $2007 \mathrm{ci}$ & 0.019 & 11.13 & 0.20 & 0.10 & $\mathrm{~Pa}$ & $\cdots$ & $\cdots$ & $\ldots$ & $\cdots$ & -3.93 & 0.19 & 0.14 & 100 \\
\hline $2008 \mathrm{~L}$ & 0.019 & 10.13 & 0.09 & 0.55 & $\mathrm{~Pa}$ & $\cdots$ & $\ldots$ & $\cdots$ & $\ldots$ & -4.26 & 0.48 & 0.19 & 100 \\
\hline 2008af & 0.034 & 11.48 & 0.09 & 0.17 & $\mathrm{~Pa}$ & -4.37 & 99.00 & 0.57 & 100 & -3.27 & 0.62 & 0.30 & 92 \\
\hline $2008 \mathrm{bf}$ & 0.026 & 11.39 & 0.28 & 0.03 & $\mathrm{~Pa}$ & -4.30 & 99.00 & 0.00 & 100 & -3.43 & 99.00 & 0.00 & 100 \\
\hline
\end{tabular}


Table 4. The weighted-mean and r.m.s. scatter of SN Ia Hubble residuals in different host environments

\begin{tabular}{|c|c|c|c|c|c|}
\hline \multirow[t]{2}{*}{ Group } & $N$ & Mean residual & Error & r.m.s. & Error \\
\hline & & (mag) & (mag) & $(\mathrm{mag})$ & $(\mathrm{mag})$ \\
\hline Locally Passive & 194 & -0.043 & 0.013 & 0.180 & 0.009 \\
\hline Locally Star-Forming & 174 & 0.038 & 0.013 & 0.172 & 0.009 \\
\hline Diff. & & 0.081 & 0.018 & & \\
\hline Globally Passive & 194 & -0.043 & 0.013 & 0.180 & 0.009 \\
\hline Globally Star-Forming & 455 & 0.006 & 0.008 & 0.167 & 0.006 \\
\hline Diff. & & 0.049 & 0.015 & & \\
\hline High-Mass $\left(\log M_{\text {stellar }}>10\right)$ & 464 & -0.022 & 0.008 & 0.172 & 0.006 \\
\hline Low-Mass $\left(\log M_{\text {stellar }} \leq 10\right)$ & 184 & 0.035 & 0.012 & 0.164 & 0.009 \\
\hline Diff. & & 0.057 & 0.014 & & \\
\hline \multicolumn{6}{|l|}{ For comparison } \\
\hline YONSEI Cosmology & 941 & 0.002 & 0.006 & 0.196 & 0.005 \\
\hline Full Local sample & 368 & -0.003 & 0.009 & 0.175 & 0.006 \\
\hline
\end{tabular}


Table 5. The best-fit flat $\Lambda \mathrm{CDM}$ parameters

\begin{tabular}{lccccccc}
\hline \hline \multicolumn{1}{c}{ Group } & $\mathrm{SNe}$ & $\Omega_{M}$ & $\alpha$ & $\beta$ & $M_{B}$ & $\sigma_{\text {int }}$ & $\chi^{2} /$ D.O.F. \\
\hline Locally Passive & 194 & $0.33_{-0.10}^{+0.09}$ & $0.18_{-0.02}^{+0.03}$ & $2.96_{-0.29}^{+0.31}$ & $-19.12_{-0.05}^{+0.04}$ & 0.104 & $190.62 / 190$ \\
Locally Star-Forming & 174 & $0.31 \pm 0.09$ & $0.14_{-0.03}^{+0.04}$ & $3.40_{-0.37}^{+0.40}$ & $-19.02 \pm 0.05$ & 0.111 & $170.58 / 170$ \\
\hline Full Local sample & 368 & $0.29_{-0.05}^{+0.07}$ & $0.14_{-0.01}^{+0.02}$ & $3.16_{-0.24}^{+0.25}$ & $-19.07 \pm 0.03$ & 0.117 & $363.60 / 364$ \\
YONSEI Cosmology & 941 & $0.31_{-0.03}^{+0.04}$ & $0.15_{-0.01}^{+0.00}$ & $3.08_{-0.15}^{+0.16}$ & $-19.06_{-0.01}^{+0.02}$ & 0.135 & $935.36 / 937$ \\
\hline
\end{tabular}

